\title{
Are Depot Clinics Out of Date?
}

\author{
Allan Beveridge, Senior Registrar, Bootham Park Hospital, York
}

Depot Clinics or 'Moderate Clinics' tend to be similar. They are usually regarded as 'low priority' and often staffed by clinical assistants with only a limited training in psychiatry. Typically a large number of patients turn up each week, and there is little time for a detailed assessment of their needs. The clinic is often seen as 'a chore' by medical and nursing staff who may feel that the long-term care and support of patients with chronic disabling illnesses lacks excitement. One gains the impression that the patients with the less attractive personalities or those presenting repetitive management problems are more likely to be referred.

The Depot Clinic at Bootham Park, serving York District, reflects some of these points. With sectorisation of psychiatric services taking place, a major review was overdue. During a six month senior registrar assignment each patient was reviewed and information was obtained about their quality of life, the nature of their illness and their contacts with the psychiatric services.

The clinic takes place on one afternoon a week and has over 90 patients on its books. Each week $30-50$ patients attend. The Depot Clinic has been largely run by clinical assistants with no formal psychiatric training. Nursing staff who administer the injections are drawn on a rota basis from the psychotherapy day hospital so their prime interest is not in the long-term management of schizophrenia.

The Patients. At the time of the survey there were 52 male patients and 39 female patients, the majority aged 30-60 years. Nearly half were single and a third married or co-habiting; the remainder were divorced or widowed. A higher proportion of females than males had partners.

A third of the patients owned their own house; $13 \%$ were living with their parents or relatives. The majority of the patients lived in lodgings, rented accommodation, hostels or group homes. A quarter lived alone.

Of those with paid employment, many were working part-time. Very few patients had demanding jobs. The most successful patient held down a permanent job as an art teacher, whilst another was a part-time university lecturer. Several patients worked in part-time clerical jobs at British Rail. This finding is similar to the recent Northwick Park study of first admission schizophrenics where only a small proportion were doing some form of meaningful work. ${ }^{1}$ Of the $81 \%$ of patients who were not working, a tenth did some form of sheltered work; $16 \%$ attended rehabilitation day units in the hospital.

Some idea of what patients actually did when they were not attending for injections was obtained. Only about a fifth attended any form of day centre. Of the remainder, some led fairly active lives and were not in need of social support. However, at least a quarter appeared to engage in very little social activity. Such patients had no social circle, had little, if any, contact with relatives and spent most of their time sitting or lying about the house. Some wandered aimlessly about the town. Two thirds of the patients did not have a community nurse or social worker. The remainder had one or both.

Clinical state. Of the patients, $91 \%$ were diagnosed as suffering from schizophrenia or paranoid psychosis. The other diagnoses included schizo-affective state, mental handicap and unspecified psychosis and epileptic psychosis. In more than half the illness had begun more than 10 years previously, and the majority had been admitted several times during the course of their illness.

During each review the patient's mental state was examined and the presence of side effects noted. Reassuringly, $85 \%$ of the patients exhibited no psychotic symptoms, and two thirds were found to have nogross physical side effects. A more structured assessment procedure would probably have uncovered more subtle manifestations of extra-pyramidal abnormalities. However, in a brief routine out-patient assessment only a third of patients had extra-pyramidal side effects. The most common was tremor of the upper limbs, and $5 \%$ of the patients were judged to have tardive dyskinesia. Although weight gain is increasingly recognised as an unwanted side effect of neuroleptic medication, only $7 \%$ of patients complained of it.

As a result of the review of each patient it was felt that a third could have their medication reduced. There are still no clear guidelines about reducing or stopping long-term intramuscular medication. Clinical decisions tend to be based on the knowledge of the patient's pattern of illness and the length of time since previous relapse. The comparatively high number of patients whose medication could be reduced probably reflects the lack of regular review that these patients have been subject to over the years. Certainly some of the patients had been on the same medication without any medical contact for several years. Only one of the patients who had a reduction in medication suffered immediate relapse, although it obviously takes several months to assess properly the response to a reduction in medication.

It is of concern that nearly half of the patients were taking oral anti-cholinergic drugs on a long-term basis. These drugs are known to affect the therapeutic effectiveness of depot medication, and perhaps also increase the chances of developing tardive dyskinesia. Many patients, however, were reluctant to have their anti-cholinergics stopped and 
complained of a variety of physical and psychological symptoms when this medication was withdrawn.

Having briefly described the running of a Depot Clinic, what are the advantages and disadvantages of this model of patient care?

Firstly the disadvantages:

1. The Depot Clinic is hospital-based. The hospital may be miles from the patient's home which can obviously affect compliance.

2. The model was organised to suit the needs of the institution-the delivery of medication to the maximum number of patients in the minimum amount of time. It is not geared primarily to the needs of the patient. Thus a large number of patients with greatly differing needs are treated in a uniform way.

3. There is little time for each patient, and the approach tends to be exclusively based on the medical model of signs, symptoms and medication. There is little facility for assessing the social environment of the patient or being able to introduce any beneficial changes to this environment. The system is crisis-based, and relatives are only seen when the patient is unwell. There is little opportunity for avoiding such crises. Since Depot Clinics were established there has been a great deal of research which has indicated that the family environment plays an important role in the course of a schizophrenic illness.

4. The staff tend to change frequently with junior medical and nursing staff rotating to other placements so there is little continuity of care. The staff do not know the patients, and the patients find it unsatisfying to see different staff when they come to the clinic. As indicated before, the medical staff often have little training in psychiatry. Staff have to rely on the case notes when reaching decisions about a patient, and the notes are often poorly documented. There may be a detailed account of the patient's social environment ten years previously when the illness began, but no record of the current situation. The clinic doctor probably knows less about the patient than the original clinical team who referred the patient.

5. With such a large group of patients it is important that there is an adequate record system concerning attendance and default. It is fairly easy to slip out of this system and be lost track of. Patients who default are often discharged, and there may be neither the time nor the enthusiasm to develop alternative strategies for such non-attenders.

The advantages:

1. The Depot Clinic is seen as a contact point when diffculties arise. Thus GPs, community nurses, relatives and patients themselves can approach the clinic. How commonly such approaches are made depends on how engaged or how enthusiastic the staff are perceived to be by the users. 2. Although the staff changes are frequent, the charge nurse at the Bootham Park Clinic has been there for five years. He provides invaluable knowledge about the patients' present social circumstances and their past history. A member of staff who has known the patient for a long time is a crucial component of any model of care for long-term patients.
3. Being hospital-based, the clinic can facilitate the admission of patients who become ill.

This is the most commonly practised model of patient care for chronic schizophrenics. There are other models which are community-based.

1. Care delivered by several smaller Mental Health Units each based in appropriate sectors of the city. The patients would have less far to travel, and the Unit would be able to develop a deeper knowledge of the local environment.

2. Care delivered by community psychiatric nurses at the patient's home. The CPN has a particular consultant team he can identify if there are difficulties. The patient has regular medical reviews.

3. Care delivered by GPs. GPs have only a small number of schizophrenics on their list. In some practices this has proved effective. However, the success of such a model depends on GPs having the time, inclination and expertise.

With the moves from hospital to the community, some form of community approach to the long-term management of schizophrenia is to be welcomed. However, there are several factors which are crucial for the success of any model of care.

1. There must be adequate basic data for each patient. A key worker should be identified, and there should be a 'tracking' system for noting non-attendance and acting appropriately.

2. Continuity of care. Ideally there should be a member of staff, either a doctor or a nurse, who has known the patient over a reasonable period of time and is aware of the patient's social environment and his previous history.

3. Adequate training and motivation of staff. Both medical and nursing staff should have psychiatric training, and their function should not be perceived as a necessary 'chore'.

\section{Conclusion}

Centrally based Depot Clinics had the advantage of economy and convenience for staff and did ensure that defaulters were identified so that action could be taken (though often it wasn't). The importance of subsequent research showing that family and other social factors are important in determining relapse means that a more personal locally based service is to be preferred. However, such dispersal of patients must be linked up with a good 'tracking system' to identify centrally when someone 'at risk' is lost touch with. At present York is in the process of moving the care of patients into the community, and Community Mental Health Centres are being developed. A computer note will go to key workers every three months for report back, and if not positively reported the team leader will be alerted to take action.

\section{ACKNOWLEDGEMENT}

I would like to thank Dr Peter Kennedy for his helpful comments about this paper.

REFERENCE

'Johnstone, E. C., Crow, T. J., Johnson, A. L. \& MacMillan, J. F. (1986) The Northwick Park study of first episodes of schizophrenia. The British Journal of Psychiatry, 148, 115-143. 